\title{
Improvement in Atrial Fibrillation-Related Symptoms After Cardioversion: Role of NYHA Functional Class and Maintenance of Sinus Rhythm
}

\author{
Maria Ferre-Vallverdu ${ }^{1-3}$ \\ Carmen Ligero ${ }^{1-3}$ \\ Rafael Vidal-Perez (iD) ${ }^{4}$ \\ Antoni Martinez-Rubio ${ }^{5}$ \\ Xavier Vinolas ${ }^{6}$ \\ Josep $M$ Alegret $^{1-3}$ \\ 'Department of Cardiology, Hospital \\ Universitari de Sant Joan; ${ }^{2}$ Institut \\ d'Investigació Sanitària Pere Virgili \\ (IISPV); ${ }^{3}$ Department of Medicine and \\ Surgery of the University Rovira i Virgili; \\ Reus, Spain; ${ }^{4}$ Department of Cardiology, \\ Complejo Hospitalario Universitario de \\ A Coruña, A Coruña, Spain; ${ }^{5}$ Department \\ of Cardiology, Hospital Parc Taulí, \\ Sabadell, Spain; ${ }^{6}$ Department of \\ Cardiology, Hospital de la Sta, Creu i St \\ Pau, Barcelona, Spain
}

\begin{abstract}
Background: The European Heart Rhythm Association (EHRA) score is a proven and validated tool for assessing the symptoms of atrial fibrillation (AF). Little is known about the variables related to this score and how it changes after cardioversion.

Methods: We analyzed 744 patients undergoing elective cardioversion in whom AF-related symptoms were assessed at baseline and after 6 months of follow-up using the EHRA score. We assessed the association between the EHRA score and other clinical and echocardiographic variables at baseline and after 6 months of follow-up.

Results: At 6 months of follow-up, we observed a reduction in the EHRA score in 50\% and worsening in $2.8 \%$ of patients who remained in sinus rhythm (SR) compared with $34.6 \%$ and $11.3 \%$, respectively, of patients with AF episodes $(\mathrm{p}<0.0001)$. Patients who maintained SR at 6 months were less symptomatic than those with AF (EHRA score $1.13 \pm 0.35$ vs $1.42 \pm$ $0.59 ; \mathrm{p}<0.0001)$. The independent predictors for reduction in the EHRA score after cardioversion were NYHA $\geq$ II at baseline and maintenance of SR $(\mathrm{p}<0.0001)$.

Conclusion: The greatest improvement in AF-related symptoms was in patients who remained in SR at 6 months after cardioversion and in patients with worse NYHA functional class at baseline.
\end{abstract}

Keywords: atrial fibrillation, EHRA score, cardioversion, NYHA functional class, sinus rhythm, symptoms improvement

\section{Introduction}

It has been widely reported that patients with atrial fibrillation (AF) have significantly poorer quality of life (QoL) than healthy controls. ${ }^{1}$ A substantial proportion of patients with AF have associated symptoms, ${ }^{2,3}$ and the severity of these symptoms has been closely correlated with impaired QoL. ${ }^{4}$

The European Heart Rhythm Association (EHRA) score has been shown to be a useful tool for assessment of AF-related symptoms and has been validated for describing symptom severity. ${ }^{5,6}$ It is simple to use and is recommended by the European Society of Cardiology guidelines for management of AF. ${ }^{7}$ The EHRA score is considered one of the strongest predictors of QoL, regardless of the type of $\mathrm{AF}$, and is commonly used to assess improvement in symptoms after treatment of $\mathrm{AF}^{8}$

Previous trials comparing rhythm and rate control strategies have reported neutral effects on stroke and mortality. ${ }^{9-11}$ However, very recently, the EAST-AFNET 4 trial has shown that early rhythm-control therapy was associated with a lower risk of
Correspondence: Josep M Alegret Department of Cardiology, Hospita Universitari de Sant Joan. Institut d'Investigació Sanitària Pere Virgili (IISPV) Department of Medicine and Surgery of the University Rovira i Virgili; Reus, Spain. C/Dr Josep Laporte, 4, Reus, 43204, Spain Email josepmaria.alegret@urv.cat 
cardiovascular outcomes. ${ }^{12}$ While both treatment strategies have been associated with improvement in QoL, ${ }^{13-15}$ it is still unclear which is superior. ${ }^{1,16}$ Cardioversion is recommended as part of rhythm control therapy in symptomatic patients with persistent or long-standing persistent $\mathrm{AF}^{7}$ However, changes symptoms related to AF after cardioversion have received little attention in the literature.

In this study, we assessed the main predictors of AFrelated symptoms based on the EHRA score at baseline and 6 months after cardioversion.

\section{Methods}

\section{Study Population}

The CARDIOVERSE study was designed to monitor the clinical practice of elective electrical cardioversion in Spain by prospectively recording all patients with persistent AF who underwent the procedure between February 1 and June 30, 2012 in 67 Spanish hospitals. $^{17}$ The study population comprised 915 patients. The inclusion criteria were age $>18$ years, duration of $\mathrm{AF}>7$ days, and absence of precipitating conditions (eg, hyperthyroidism, fever, cardiac surgery, and pericarditis). Pre-procedural antiarrhythmic drugs could be prescribed at the discretion of the physician. Those patients treated with antiarrhythmic drugs who reverted to $\mathrm{SR}$ previous to electrical cardioversion were considered who had a pharmacological cardioversion. Patients were followed for 1 year (visits at 1, 3, 6, and 12 months after cardioversion).

AF-related symptoms assessment according to the EHRA classification was planned in the design of the CARDIOVERSE study. In this report, we selected retrospectively 744 patients with a complete record of the EHRA score before cardioversion and after 6 months of follow-up. Patients who underwent pulmonary vein ablation during follow-up were excluded.

The study was conducted according to the principles of the Declaration of Helsinki and was approved by the local ethic committees (Hospital Universitari Sant Joan de Reus 11-12-22/12obs3). Written informed consent was obtained from all the patients who participated in this study.

\section{Evaluation of Symptoms}

The physician categorized AF-related symptoms according to the EHRA classification as class I (no symptoms), class II (mild symptoms: normal daily activity not affected), class III (severe symptoms: normal daily activity affected), or class IV (disabling symptoms: normal daily activity discontinued). The EHRA score was recorded before cardioversion and after 6 months of follow-up. The NYHA symptom classification was also recorded at the beginning of the study.

Maintenance of sinus rhythm (SR) during follow-up was defined as the absence of recurrence of AF at visit 1 , visit 3, and visit 6 .

\section{Statistical Analysis}

Normally distributed continuous data are presented as mean $\pm \mathrm{SD}$. Categorical variables are presented as frequencies and percentages.

Differences in continuous variables between categories of patients were studied using an unpaired $t$-test or ANOVA, where applicable. A paired $t$-test was used to analyze the differences in the mean EHRA score between the initial score and at 6 months of follow-up.

Differences in proportions were analyzed using the chisquare test. Correlations between the NYHA classification and the EHRA score were assessed using Spearman correlation coefficient. Univariable and multivariable regression analysis were performed to assess predictors of being symptomatic (EHRA score $\geq$ II) at baseline (logistic regression) and to characterize predictors of a change in EHRA score 6 months after cardioversion (linear regression).

All statistical tests were performed using IBM SPSS Statistics for Windows, Version 20.0. A 2-tailed $\mathrm{p}$ value $<$ 0.05 was considered statistically significant.

\section{Results}

\section{Patient Characteristics}

Cardioversion was successful in 624 of the 744 patients included (83.9\%: electrical cardioversion, $\mathrm{n}=560$ [75.3\%]; pharmacological cardioversion, $\mathrm{n}=64, \quad[8.6 \%])$. Cardioversion failed in the remaining 120 patients $(16.1 \%)$.

The mean age was 63 years, and $77 \%$ were male. The most common comorbidity was hypertension (59.9\%), and $29.8 \%$ of patients had structural heart disease. Baseline clinical characteristics according to the EHRA score are presented in Table 1. The clinical characteristics of the patients excluded because their EHRA score was not available were similar to those of the patients included in this analysis (Supplementary Table 1). 
Table I Baseline Characteristics According to EHRA Score

\begin{tabular}{|c|c|c|c|c|c|}
\hline & $N=744$ & EHRA I (3II) & EHRA II (363) & EHRA III/IV (70) & $\mathbf{p}$ \\
\hline Age & $63 \pm 10$ & $62 \pm 9$ & $64 \pm 10$ & $65 \pm 11$ & 0.004 \\
\hline Female & $169(22.7 \%)$ & 45 (I4.5\%) & $100(27.5 \%)$ & 24 (34.3\%) & $<0.0001$ \\
\hline DM & 143 (19.2\%) & 48 (I5.4\%) & 77 (2I.2\%) & 18 (25.7\%) & 0.06 \\
\hline Hypertension & 446 (59.9\%) & $169(54.3 \%)$ & $237(65.3 \%)$ & 40 (57.1\%) & 0.013 \\
\hline COPD & 77 (10.3\%) & $25(8 \%)$ & 44 (I2.1\%) & 8 (1 I.4\%) & 0.212 \\
\hline Structural heart disease & $222(29.8 \%)$ & 61 (19.6\%) & $125(34.4 \%)$ & $36(5 \mathrm{I} .4 \%)$ & $<0.0001$ \\
\hline Prior stroke & $4 \mathrm{l}(5.5 \%)$ & $14(4.5 \%)$ & $25(6.9 \%)$ & $2(2.9 \%)$ & 0.237 \\
\hline \multicolumn{6}{|l|}{ Duration of $\mathrm{AF}$} \\
\hline Persistent AF & $509(68.4 \%)$ & 185 (59.5\%) & $27 \mid(74.6 \%)$ & $53(75.7 \%)$ & \multirow[t]{3}{*}{$<0.0001$} \\
\hline Long-standing persistent AF (>I year) & $132(17.7 \%)$ & 69 (22.2\%) & 55 (I5.2\%) & 8 (1 $1.4 \%)$ & \\
\hline Unknown & $103(13.8 \%)$ & $57(18.3 \%)$ & 37 (10.2\%) & $9(12.9 \%)$ & \\
\hline \multicolumn{6}{|l|}{$\mathrm{CHA}_{2} \mathrm{DS}_{2} \mathrm{VASC}$} \\
\hline$<2$ & 304 (40.9\%) & $160(51.4 \%)$ & $125(34.4 \%)$ & 19 (27.1\%) & \multirow[t]{2}{*}{$<0.0001$} \\
\hline$\geq 2$ & $440(59.1 \%)$ & I5I (48.6\%) & $238(65.6 \%)$ & $5 \mathrm{I}(72.9 \%)$ & \\
\hline \multicolumn{6}{|l|}{ HASBLED } \\
\hline$<2$ & $555(74.6 \%)$ & $243(78.1 \%)$ & $265(73 \%)$ & $47(66.1 \%)$ & \multirow[t]{2}{*}{0.101} \\
\hline$\geq 2$ & $189(25.4 \%)$ & $68(21.9 \%)$ & $98(27 \%)$ & $23(32.9 \%)$ & \\
\hline \multicolumn{6}{|l|}{ NYHA classification } \\
\hline I & $648(87.1 \%)$ & 307 (98.7\%) & $297(81.8 \%)$ & $44(62.9 \%)$ & \multirow[t]{2}{*}{$<0.0001$} \\
\hline II, III, IV & $96(12.9 \%)$ & $4(1.3 \%)$ & $66(18.2 \%)$ & $26(37.1 \%)$ & \\
\hline LVEF (\%) & $57 \pm 11$ & $59 \pm 9$ & $56 \pm 12$ & $49 \pm 16$ & $<0.0001$ \\
\hline LA diameter & $44 \pm 6$ & $44 \pm 6$ & $43 \pm 6$ & $46 \pm 6$ & 0.032 \\
\hline \multicolumn{6}{|l|}{ Antiarrhythmic therapy } \\
\hline Flecainide/propafenone & 77 (10.3\%) & $4 \mathrm{I}(13.2 \%)$ & 32 (8.8\%) & $4(5.7 \%)$ & \multirow[t]{3}{*}{0.593} \\
\hline Amiodarone & $216(29 \%)$ & $83(26.7 \%)$ & $116(32 \%)$ & 17 (24.3\%) & \\
\hline Other & $19(2.6 \%)$ & 9 (2.9\%) & 9 (2.5\%) & I (I.4\%) & \\
\hline \multicolumn{6}{|l|}{ Other treatment } \\
\hline Betablockers & 465 (62.5\%) & I8I (58.2\%) & $234(64.5 \%)$ & 50 (7I.4\%) & 0.066 \\
\hline Calcium blockers & I33 (I7.9\%) & $56(18 \%)$ & 67 (I8.5\%) & $10(14.3 \%)$ & 0.704 \\
\hline Digoxin & $76(10.2 \%)$ & $23(7.4 \%)$ & 42 (II.6\%) & II (I5.7\%) & 0.057 \\
\hline ACEi/ARB & $390(52.4 \%)$ & $150(48.2 \%)$ & $199(54.8 \%)$ & $4 \mathrm{l}(58.6 \%)$ & 0.129 \\
\hline
\end{tabular}

Abbreviations: ACEi, angiotensin-converting enzyme inhibitor; AF, atrial fibrillation; ARB, angiotensin receptor blocker; COPD, chronic obstructive pulmonary disease; DM, diabetes mellitus; LA; left atrium; LVEF, left ventricular ejection fraction; NYHA, New York Heart Association. 


\section{Symptom Burden at Baseline}

At enrolment, 311 patients $(41.8 \%)$ were classified as having no AF-related symptoms (EHRA I). The remaining patients were classified as having mild symptoms (EHRA II, 48.8\%), severe symptoms (EHRA III, 8.2\%), and disabling symptoms (EHRA IV, 1.2\%). Compared with asymptomatic patients (EHRA I), symptomatic patients (EHRA score $\geq$ II) were older ( $64 \pm 10$ vs $62 \pm 9$ years; $\mathrm{p}=0.001)$ and more likely to be female $(28.6 \%$ vs $14.5 \%$; $\mathrm{p}<0.0001)$. They also had a greater prevalence of structural heart disease $(37.2 \%$ vs $19.6 \%$; $<0.0001)$, diabetes mellitus $(21.9 \%$ vs $15.4 \% ; \mathrm{p}=0.026)$, and hypertension (64\% vs $54.3 \% ; \mathrm{p}=0.008)$, as well as a higher NYHA classification $(21.2 \%$ in class II-IV vs $1.3 \%$; $p<0.0001)$ and lower left ventricular ejection fraction (LVEF) (55 \pm 13 vs $59 \pm 9 ; \mathrm{p}<0.0001)$. The independent predictors for being symptomatic (EHRA score II-IV) at baseline were female gender $(\mathrm{OR}=2.22 ; 95 \% \mathrm{CI}, 1.44-3.42 ; \mathrm{p}<$ 0.0001 ), and the strongest predictor was being in NYHA class II-IV $(\mathrm{OR}=12.5 ; 95 \% \mathrm{CI}, 4.4-35.4 ; \mathrm{p}<0.0001)$. (Supplementary Table 2)

We observed a significant correlation when comparing baseline EHRA score with NYHA score $(\mathrm{R}=0.334$, $\mathrm{p}<$ $0.0001)$. The correlation was more pronounced in patients with $L V E F \leq 40 \%(\mathrm{R}=0.506, \mathrm{p}<0.0001)$ than in patients with LVEF $>40 \%(\mathrm{R}=0.264, \mathrm{p}<0.0001)$.

\section{Predictors of Improvement in AF-Related Symptoms at 6 Months}

During follow-up, 282 patients (37.9\%) maintained SR. In the cohort that maintained SR, mean EHRA score improved during follow-up $(1.13 \pm 0.35)$ with respect to baseline $(1.67 \pm 0.63),(\mathrm{p}<0.0001)$. However, in patients who did not maintain SR, symptoms also improved during follow-up $(1.42 \pm 0.59$ at 6 months vs $1.7 \pm 0.7$ at baseline; $\mathrm{p}<0.0001)$. Otherwise, patients who maintained SR were less symptomatic at 6 months than patients whose $\mathrm{AF}$ relapsed $(1.13 \pm 0.35$ vs $1.42 \pm 0.59 ; \mathrm{p}<0.0001)$ despite having similar symptoms at baseline $(1.67 \pm 0.63$ vs $1.7 \pm 0.7 ; \mathrm{p}=0.57$ ). Figure 1 .

Six months after cardioversion, the EHRA score decreased in 301 patients $(40.4 \%)$, remained unchanged in 383 patients $(51.5 \%)$, and worsened in $60(8.1 \%)$. When patients were stratified according to maintenance of SR, the EHRA score fell in 50\% of those who maintained SR compared with $34.6 \%$ of those who did not maintain SR, while no change in EHRA score was observed in $47.2 \%$ of

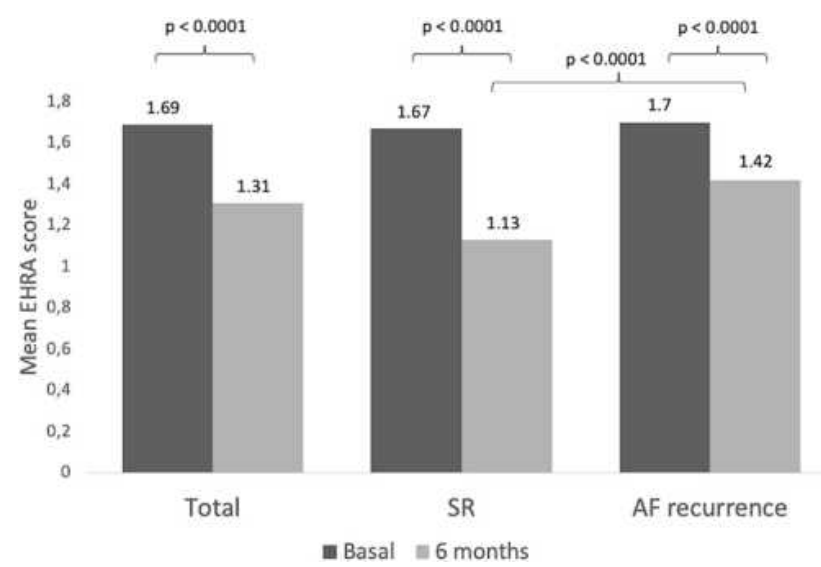

Figure I Mean EHRA score at baseline and after 6 months of follow-up in the study population, in patients who maintained sinus rhythm, and in patients who experienced recurrence of $\mathrm{AF}$.

patients with SR compared with $54.1 \%$ of those whose AF relapsed. The EHRA score increased in $2.8 \%$ of patients in SR compared with $11.3 \%$ of those whose AF relapsed $(\mathrm{p}<$ 0.0001). Figure 2 .

In the univariable analysis, the factors associated with a change in EHRA score at 6 months were structural heart disease, baseline NYHA classification $\geq 2$, LVEF, and maintenance of SR. In the multivariable linear analysis to predict change in EHRA score at 6 months after cardioversion, the only statistically significant variables were NYHA classification $\geq 2(\beta=-0.178 ; p<0.0001)$ and maintenance of SR $(\beta=-0.162 ; p<0.0001)$. (Table 2)

\section{Discussion}

In the present study, we identified variables related to the EHRA score at baseline and to improvement in the EHRA score after cardioversion. We highlight that maintenance of SR at 6 months was related to an improved EHRA score. We also highlight the influence of the NYHA as a predictor of improvement in the EHRA score, a higher NYHA functional class at baseline was related to a more pronounced decrease in EHRA score at 6 months.

AF-related symptoms diminished during follow-up in the group as a whole, probably indicating greater tolerance and better control of heart rate. However, we observed few symptoms in those who remained in SR, thus indicating that maintenance of SR was the strongest independent predictor of an improvement in AF-related symptoms. The role of maintaining SR in improvement in symptoms remains controversial. ${ }^{18-20}$ A recent subanalysis of the CABANA trial showed that higher rates of maintenance 


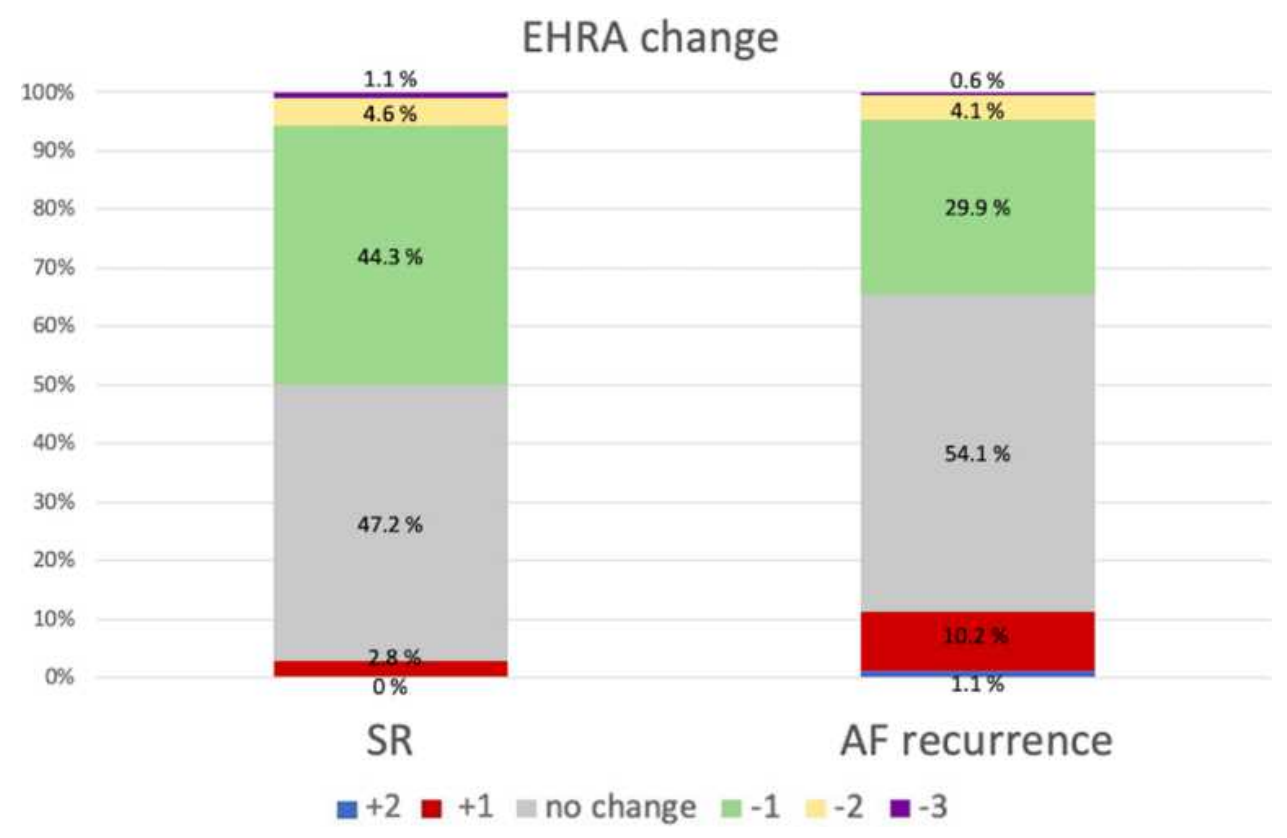

Figure 2 Change in EHRA at 6 months after cardioversion in patients who maintained sinus rhythm and in patients with recurrence of AF.

of SR achieved using AF ablation were related to a significant improvement in QoL. ${ }^{21}$ On the other hand, a report from the ORBIT-AF registry which compared matched cardioverted and non-cardioverted patients, showed an improvement in AF-related symptoms and quality of life with both strategies. ${ }^{22}$ However, in that nonrandomized study, which included all types of AF, maintenance of SR during follow-up was not considered in the analysis. Given our results and these observations, individualized decisions should be based on AF-related symptoms, and the probable benefit of maintaining SR should be taken into consideration. Therefore, in many cases, it may be necessary to consider complementing the rhythm

Table 2 Univariable and Multivariable Linear Regression Analysis for Prediction of Change in EHRA Score

\begin{tabular}{|l|c|c|c|c|}
\hline \multirow{2}{*}{} & \multicolumn{2}{|c|}{ Univariable } & \multicolumn{2}{c|}{ Multivariable } \\
\cline { 2 - 5 } & $\beta$ & $\mathbf{p}$ value & $\beta$ & p value \\
\hline Age (years) & -0.012 & 0.74 & - & - \\
Female sex & -0.07 & 0.055 & $-0.6 \mathrm{I}$ & $0, \mathrm{I}$ \\
Structural heart disease & -0.112 & 0.002 & -0.23 & 0.584 \\
NYHA classification $\geq 2$ & -0.214 & $<0.000 \mathrm{I}$ & -0.178 & $<0.000 \mathrm{I}$ \\
LVEF & -0.146 & $<0.000 \mathrm{I}$ & -0.065 & 0,128 \\
Maintenance of SR & -0.17 & $<0.000 \mathrm{I}$ & -0.162 & $<0.000 \mathrm{I}$ \\
Antiarrhythmic treatment & -0.096 & 0.09 & - & - \\
\hline
\end{tabular}

Abbreviations: LVEF, left ventricular ejection fraction; NYHA, New York Heart Association; SR, sinus rhythm. control strategy with cardioversion followed by pulmonary vein ablation.

The many variables that may influence the EHRA score have been reported in different registries and include female gender, coronary artery disease, heart failure, AF type, chronic obstructive pulmonary disease, sleep apnea, limited physical activity, and poor sleep quality. ${ }^{2-4,23}$ The relationship between heart failure and AF has been extensively investigated in the literature, which shows that both diseases are linked by similar risk factors, share a common pathophysiology, ${ }^{24,25}$ and frequently present with similar symptoms. The NYHA scale has been widely used to assess symptoms of heart failure, although, to the best of our knowledge, the relationship between the NYHA scale classification and the EHRA score had not been previously evaluated. In our study, we observed a direct correlation between baseline NYHA functional class and the EHRA scale, suggesting that the greater the deterioration of the functional class, the worse the tolerance to AF. This correlation was even stronger in patients with reduced LVEF, probably owing to the well-documented heterogeneity in heart failure with preserved LVEF. At the same time, after cardioversion, we observed a greater symptomatic benefit related to $\mathrm{AF}$ in patients with a worse baseline NYHA functional class; those with a worse functional class at baseline were those who had a greater opportunity to improve during follow-up. On the other hand, there is likely to be an overlap in the patient's interpretation of 
heart failure and symptoms of AF, as dyspnea on exertion is one of the main symptoms of $\mathrm{AF}^{4}$

We found that patients who were symptomatic at baseline (EHRA II-IV) were older and more likely to be female. Other reports point to older age as a significant predictor of asymptomatic AF. ${ }^{2}$ However, we previously reported the predominance of older men undergoing cardioversion. Our results suggest a trend toward selecting older and more symptomatic patients for cardioversion.

Although current recommendations state that rhythm control therapy is indicated to improve symptoms, ${ }^{7}$ we were surprised by the high proportion of asymptomatic patients $(41.8 \%)$ who underwent cardioversion. Other studies have reported a high proportion of asymptomatic patients undergoing AF ablation. ${ }^{26}$ This may reflect the perception among clinicians that rhythm control therapy has favorable effects on cardiovascular hemodynamics.

\section{Limitations}

Around a quarter of the patients included in this study (19\%) did not have an EHRA score at enrolment or during follow-up. However, no significant differences in clinical characteristics were found with respect to those included in the analysis.

While a modified EHRA score is now available, it had not been developed at the time this study was performed. In any case, the classic EHRA score continues to be the most widely used.

Although it might seem obvious that the patients who maintained SR at 6 months had a score of 1 in the EHRA score at 6 months, we must consider that the patients could have had a certain burden of AF during this period that was reflected in their assessment. However, a certain overlap of the symptoms that the patient could attribute to AF with other pathologies such as heart failure cannot be ruled out.

\section{Conclusions}

In this cohort of patients undergoing elective cardioversion, those who maintained SR after 6 months of follow-up experienced the most marked improvement in AF-related symptoms. NYHA functional class was directly related to the improvement in the EHRA score after cardioversion. These findings should encourage us to investigate appropriate strategies for maintaining SR after cardioversion.

\section{Funding}

This work was supported by Bayer Health Care Spain. "The funders had no role in study design, data collection and analysis, decision to publish, or preparation of the manuscript." "All authors made substantial contributions to conception and design, acquisition of data, or analysis and interpretation of data; took part in drafting the article or revising it critically for important intellectual content; agreed to submit to the current journal; gave final approval of the version to be published; and agree to be accountable for all aspects of the work."

\section{Disclosure}

The authors report no conflicts of interest in this work.

\section{References}

1. Thrall G, Lane D, Carroll D, Lip GYH. Quality of life in patients with atrial fibrillation: a systematic review. Am J Med. 2006;119:448e1. doi:10.1016/j.amjmed.2005.10.057

2. Boriani G, Laroche C, Diemberger I, et al. Asymptomatic atrial fibrillation: clinical correlates, management, and outcomes in the EORP-AF pilot general registry. Am J Med. 2015;128:509-518. doi:10.1016/j.amjmed.2014.11.026

3. Bakhai A, Darius H, De Caterina R, et al. Characteristics and outcomes of atrial fibrillation patients with or without specific symptoms: results from the PREFER in AF registry. Eur Hear J - Qual Care Clin Outcomes. 2016;2:299-305. doi:10.1093/ehjqcco/qcw031

4. Freeman JV, Simon DN, Go AS, et al. Association between atrial fibrillation symptoms, quality of life, and patient outcomes: results from the outcomes registry for better informed treatment of atrial fibrillation (ORBIT-AF). Circ Cardiovasc Qual Outcomes. 2015;8:393-402. doi:10.1161/CIRCOUTCOMES.114.001303

5. Kirchhof P, Auricchio A, Bax J, et al. Outcome parameters for trials in atrial fibrillation: recommendations from a consensus conference organized by the German atrial fibrillation competence NETwork and the European heart rhythm association. Europace. 2007;9:1006-1023. doi:10.1093/europace/eum191

6. Wynn GJ, Todd DM, Webber M, et al. The European Heart Rhythm Association symptom classification for atrial fibrillation: validation and improvement through a simple modification. Europace. 2014;16:965-972. doi:10.1093/europace/eut395

7. Kirchhof P, Benussi S, Kotecha D, et al. 2016 ESC Guidelines for the management of atrial fibrillation developed in collaboration with EACTS. Eur Heart J. 2016;37:2893-2962.

8. Witassek F, Springer A, Adam L, et al. Health-related quality of life in patients with atrial fibrillation: the role of symptoms, comorbidities, and the type of atrial fibrillation. PLoS One. 2019;14:1-14. doi:10.1371/journal.pone.0226730

9. Chatterjee S, Sardar P, Lichstein E, Mukherjee D, Aikat S. Pharmacologic rate versus rhythm-control strategies in atrial fibrillation: an updated comprehensive review and meta-analysis. PACE Pacing Clin Electrophysiol. 2013;36:122-133. doi:10.1111/j.15408159.2012.03513.x

10. Al-Khatib SM, Allen LaPointe NM, Chatterjee R, et al. Rate- and rhythm-control therapies in patients with atrial fibrillation: a systematic review. Ann Intern Med. 2014;160:760-773. doi:10.7326/M13-1467

11. Packer DL, Mark DB, Robb RA, et al. Effect of catheter ablation vs antiarrhythmic drug therapy on mortality, stroke, bleeding, and cardiac arrest among patients with atrial fibrillation: the CABANA randomized clinical trial. JAMA - J Am Med Assoc. 2019;321:1261-1274. doi:10.1001/jama.2019.0693 
12. Kirchhof P, Camm AJ, Goette A, et al. Early rhythm-control therapy in patients with atrial fibrillation. $N$ Engl $J$ Med. 2020;383:1305-1316. doi:10.1056/NEJMoa2019422

13. Grönefeld GC, Lilienthal J, Kuck KH, Hohnloser SH. Impact of rate versus rhythm control on quality of life in patients with persistent atrial fibrillation: results from a prospective randomized study. Eur Heart J. 2003;24:1430-1436. doi:10.1016/S0195-668X(03)00261-6

14. Pepine CJ. Effects of pharmacologic therapy on health-related quality of life in elderly patients with atrial fibrillation: a systematic review of randomized and nonrandomized trials. Clin Med Insights Cardiol. 2013;7:1-20. doi:10.4137/CMC.S10628

15. Jenkins LS, Brodsky M, Schron E, et al. Quality of life in atrial fibrillation: the atrial fibrillation follow-up investigation of rhythm management (AFFIRM) study. Am Heart J. 2005;149:112-120.

16. Rienstra M, Lubitz SA, Mahida S, et al. Symptoms and functional status of patients with atrial fibrillation: state of the art and future research opportunities. Circulation. 2012;125:2933-2943. doi:10.1161/CIRCULATIONAHA.111.069450

17. Alegret JM, Viñolas X, Martínez-Rubio A, et al. Gender differences in patients with atrial fibrillation undergoing electrical cardioversion. J Women's Heal. 2015;24:466-470. doi:10.1089/jwh.2014.5014

18. Ha ACT, Breithardt G, Camm AJ, et al. Health-related quality of life in patients with atrial fibrillation treated with rhythm control versus rate control: insights from a prospective international registry (registry on cardiac rhythm disorders assessing the control of atrial fibrillation: REC. Circ Cardiovasc Qual Outcomes. 2014;7:896-904. doi:10.1161/HCQ.0000000000000011

19. Hagens VE, Ranchor AV, Van Sonderen E, et al. Effect of rate or rhythm control on quality of life in persistent atrial fibrillation: results from the rate control versus electrical cardioversion (RACE) study. J Am Coll Cardiol. 2004;43:241-247. doi:10.1016/j.jacc.2003.08.037
20. Steg PG, Alam S, Chiang C-E, et al. Symptoms, functional status and quality of life in patients with controlled and uncontrolled atrial fibrillation: data from the RealiseAF cross-sectional international registry. Heart. 2012;98(3):195-201. doi:10.1136/heartjnl-2011300550

21. Mark DB, Anstrom KJ, Sheng S, et al. Effect of catheter ablation vs medical therapy on quality of life among patients with atrial fibrillation: the CABANA randomized clinical trial. JAMA - J Am Med Assoc. 2019;321:1275-1285. doi:10.1001/jama.2019.0692

22. Pokorney SD, Kim S, Thomas L, Fonarow GC, Kowey PR. Cardioversion and subsequent quality of life and natural history of atrial fibrillation. Am Heart J. 2017;185:59-66. doi:10.1016/j. ahj.2016.10.018

23. Szymanski FM, Filipiak KJ, Karpinski G, Platek AE, Opolski G. Occurrence of poor sleep quality in atrial fibrillation patients according to the EHRA score. Acta Cardiol. 2014;69:291-296. doi:10.1080/ AC.69.3.3027832

24. Kotecha D, Chudasama R, Lane DA, Kirchhof P, Lip GYH. Atrial fibrillation and heart failure due to reduced versus preserved ejection fraction: a systematic review and meta-analysis of death and adverse outcomes. Int $J$ Cardiol. 2016;15:660-666. doi:10.1016/j. ijcard.2015.10.220

25. Kotecha D, Piccini JP. Atrial fibrillation in heart failure: what should we do? Eur Heart J. 2015;36:3250-3257. doi:10.1093/eurheartj/ ehv513

26. Björkenheim A, Brandes A, Magnuson A, et al. Assessment of atrial fibrillation-specific symptoms before and 2 years after atrial fibrillation ablation: do patients and physicians differ in their perception of symptom relief? JACC Clin Electrophysiol. 2017;3:1168-1176. doi:10.1016/j.jacep.2017.04.003
Clinical Interventions in Aging

\section{Publish your work in this journal}

Clinical Interventions in Aging is an international, peer-reviewed journal focusing on evidence-based reports on the value or lack thereof of treatments intended to prevent or delay the onset of maladaptive correlates of aging in human beings. This journal is indexed on PubMed Central, MedLine, CAS, Scopus and the Elsevier

\section{Dovepress}

Bibliographic databases. The manuscript management system is completely online and includes a very quick and fair peer-review system, which is all easy to use. Visit http://www.dovepress.com/ testimonials.php to read real quotes from published authors. 